\title{
Verzeichnis abgekürzter Literaturangaben und benutzter Thukydidesausgaben
}

\author{
Stefano Valente
}

\begin{tabular}{|c|c|c|}
\hline Adler, RE & $=$ & Ada A., Suidas [1], RE IV A/1, 1931, 675-717 \\
\hline Adler, Proll. / Suidas & $=$ & $\begin{array}{l}\text { Ada A., Prolegomena, in: Suidae Lexicon, ed. Ad } \\
\text { A., I, Lipsiae 1928, V-XXIV }\end{array}$ \\
\hline lberti & $=$ & siehe Thukydidesausgaben: Alberti \\
\hline lberti, Noterelle & $=$ & $\begin{array}{l}\text { Giovan B. A., Noterelle tucididee, A\&R 25, } 1980 \\
15-22\end{array}$ \\
\hline lberti, Praef. I, II & $=$ & siehe Thukydidesausgaben: Alberti \\
\hline , VII & $=$ & $\begin{array}{l}\text { Giovan B. A., Que } \\
\text { testo. VI-VII, BPE }\end{array}$ \\
\hline lberti, Quest. VIII & $=$ & $\begin{array}{l}\text { Giovan B. A., Ques } \\
\text { testo. VIII, BPEC } 9\end{array}$ \\
\hline lberti, Quest. IX & $=$ & $\begin{array}{l}\text { Giovan B. A., Questioni tucidi } \\
\text { testo. IX, BPEC 10, 1962, 27-3 }\end{array}$ \\
\hline lberti, Quest. X & $=$ & $\begin{array}{l}\text { Giovan B. A., Quest } \\
\text { testo. X-XI, BPEC }\end{array}$ \\
\hline llberti, Ques & & $\begin{array}{l}\text { Giovan B. A., Questioni Tucididee. Per la storia del } \\
\text { testo. XIII-XIV, BPEC 15, 1967, 3-16 }\end{array}$ \\
\hline Alpers, Bericht & $=$ & $\begin{array}{l}\text { Klaus A., Bericht über Stand und Methode der Aus- } \\
\text { gabe des Etymologicum Genuinum (mit einer Aus- } \\
\text { gabe des Buchstaben } \Lambda \text { ), København } 1969\end{array}$ \\
\hline Alpers, BZ 64, 1971 & $=$ & 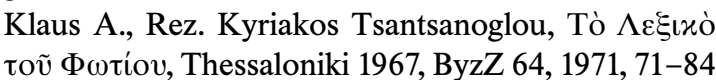 \\
\hline $\begin{array}{l}\text { Alpers, Eine } \\
\text { byzantinische }\end{array}$ & & $\begin{array}{l}\text { Klaus A., Eine byzantinische Enzyklopädie des } \\
\text { 9. Jahrhunderts. Zu Hintergrund, Entstehung und }\end{array}$ \\
\hline & & $\begin{array}{l}\text { Geschichte des griechischen Etymologikons in } \\
\text { Konstantinopel und im italogriechischen Bereich, } \\
\text { in: Guglielmo Cavallo-Giuseppe De Gregorio-Ma-M- } \\
\text { rilena Maniaci (edd.), Scritture, libri e testi nelle }\end{array}$ \\
\hline $\begin{array}{l}\text { Alpers, Klassische } \\
\text { Philologie }\end{array}$ & & $\begin{array}{l}\text { Klaus A., Klassische Philologie in Byzanz [Rez. Ni- } \\
\text { gel G. Wilson, Scholars of Byzantium, Baltimore } \\
\text { 1983], CPh 83, 1988, 342-360 }\end{array}$ \\
\hline & & \\
\hline
\end{tabular}


Alpers, Oros

Alpers, RE / Zonaras

Alpers, Theognost

Bartoletti

Beckby

Beobachtungen I

Beobachtungen II

Beobachtungen III

Beobachtungen zu einigen recc.

Beutler, Porphyrios

Bibliotheca critica nova

Cavalieri-Lietzmann, Specimina

Cobet, Hypereides ${ }^{2}$

Cunningham, Introduction / Synag. / Synagoge

Devreesse

Dover

Erbse, Bd. II / Bd. III

Erbse, Beiträge

Erbse, Untersuchungen
Mittelalter. Dargestellt an ausgewählten Beispielen, in: Hans-Albert Koch (Hrsg.), Welt der Information. Wissen und Wissensvermittlung in Geschichte und Gegenwart, Stuttgart 1990, 14-38

= Das attizistische Lexikon des Oros, Untersuchungen und kritische Ausgabe der Fragmente v. Klaus A., Berlin-New York 1981

= Klaus A., „Zonarae“ Lexicon, RE X A, 1972, 732-763

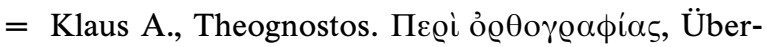
lieferung, Quellen und Text der Kanones 1-84, Diss. Hamburg 1964

= Vittorio B., Per la storia del testo di Tucidide, Firenze 1937

= Anthologia Graeca, I. Buch I-VI, GriechischDeutsch ed. Hermann B., München 1957

$=$ Philologus 1964: siehe Kleinlogel

= Philologus 142 / Philologus 1998: siehe Kleinlogel

$=$ Philologus 2011: siehe Kleinlogel

$=$ siehe Kleinlogel

= Rudolf B., Porphyrios [21], RE XXII/1, 1953, 275-313

= Bibliotheca critica nova, edd. Ianus Bake-Iacobus Geel-Hendricus A. Hamaker-Petrus Hofman Peerlkamp, II, Lugduni Batavorum 1826

= Pio Franchi de' C.-Hans L., Specimina codicum Graecorum Vaticanorum, Bonnae 21929

= Carel G. C. (Hg.), Hyperidis orationes duae. ${ }^{\circ} \mathrm{O} \varepsilon \dot{\varepsilon} \mathrm{\tau}-$

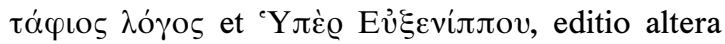
auctior et emendatior, Lugduni Batavorum 1877

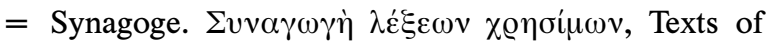
the Original Version and of MS. B, Ian C. C., Berlin-New York 2003

$=$ Robert D., Introduction à l'étude des manuscrits grecs, Paris 1954

= Kenneth J. D., The Patmos Scholia and the Text of Thucydides, CR N. S. 5, 1955, 134-137

$=$ Hartmut E., Scholia Graeca in Homeri Iliadem, Vol. II / Vol. III, Berlin 1971. 1974

= Harmut E., Beiträge zur Überlieferung der Iliasscholien, München 1960

= Harmut E., Untersuchungen $\mathrm{zu}$ den attizistischen Lexika, Berlin 1950 


\begin{tabular}{|c|c|c|}
\hline Fabricius, Bibl. & $=$ & $\begin{array}{l}\text { Johann A. F., Bibliotheca Graeca [...], II, Hamburgi } \\
{ }^{4} 1791\end{array}$ \\
\hline Gesch. & $=$ & siehe Kleinlogel \\
\hline Gnomon 1977 & $=$ & Rez. Alberti I: siehe Kleinlogel \\
\hline Gomme & $=$ & $\begin{array}{l}\text { Arnold W. G., A Historical Commentary on Thucy- } \\
\text { dides, I, Oxford 1945; II, Oxford 1956; III, Oxford } \\
1956\end{array}$ \\
\hline Hedberg & $=$ & $\begin{array}{l}\text { Torsten H., Eustathios als Attizist, Diss. Uppsala } \\
1935\end{array}$ \\
\hline Heimannsfeld & $=$ & $\begin{array}{l}\text { Heinrich H., De Helladii Chrestomathia quaestio- } \\
\text { nes selectae, Diss. Bonn } 1911\end{array}$ \\
\hline Hemmerdinger, Essai & $=$ & $\begin{array}{l}\text { Bertrand H., Essai sur l'histoire du texte de Thucy- } \\
\text { dide, Paris } 1955\end{array}$ \\
\hline Hermann, De em. rat. & $=$ & $\begin{array}{l}\text { Gottfried H., De emendanda ratione Graecae gram- } \\
\text { maticae. Pars prima. Accedunt Herodiani aliorum- } \\
\text { que libelli nunc primum editi, Lipsiae } 1801\end{array}$ \\
\hline Hilgard, Praef. & $=$ & $\begin{array}{l}\text { Theodosii Alexandrini canones, Georgi Choero- } \\
\text { bosci scholia, Sophronii Patriarchae Alexandrini ex- } \\
\text { cerpta, rec. et app. crit. indicesque adi. Alfredus H., } \\
\text { IV, Prolegomena, Lipsiae } 1894\end{array}$ \\
\hline Hornblower & $=$ & $\begin{array}{l}\text { Simon H., A Commentary on Thucydides, I, Ox- } \\
\text { ford 1991; II, Oxford } 1996\end{array}$ \\
\hline Hude, Praef. & $=$ & $\begin{array}{l}\text { siehe Thukydidesausgaben: Hude (Scholia in Thu- } \\
\text { cydidem) }\end{array}$ \\
\hline Hunger, Literatur & $=$ & $\begin{array}{l}\text { Herbert H., Die hochsprachliche profane Literatur } \\
\text { der Byzantiner, I, München } 1978\end{array}$ \\
\hline $\begin{array}{l}\text { Irigoin, Les manuscrits } \\
\text { grecs / Lustrum }\end{array}$ & $=$ & $\begin{array}{l}\text { Jean I., Les manuscrits grecs } 1931-1960 \text {, Lustrum 7, } \\
\text { 1962, 5-93 }\end{array}$ \\
\hline $\begin{array}{l}\text { Irigoin, Les manuscrits } \\
\text { d'historiens }\end{array}$ & $=$ & $\begin{array}{l}\text { Jean I., Les manuscrits d'historiens grecs et byzan- } \\
\text { tins à } 32 \text { lignes, in: Kurt Treu (Hrsg.), Studia Codi- } \\
\text { cologica, Berlin 1977, 237-245 }\end{array}$ \\
\hline Irigoin, Pour une étude & $=$ & $\begin{array}{l}\text { Jean I., Pour une étude des centres de copie byzan- } \\
\text { tins. II, Scriptorium 13, 1959, 177-209 }\end{array}$ \\
\hline Irigoin, $\mathrm{RPh} 40$ & $=$ & Jean I., Rez. Gesch., RPh 40, 1966, 312-314 \\
\hline Kleinlogel & $=$ & $\begin{array}{l}\text { Beobachtungen zu den Thukydidesscholien I, Philo- } \\
\text { logus 108, 1964, 233-246 (= Beobachtungen I; Phi- } \\
\text { lologus 1964) } \\
\text { Beobachtungen zu den Thukydidesscholien II, Phi- } \\
\text { lologus 142, 1998, 11-40 (= Beobachtungen II; Phi- } \\
\text { lologus 142; Philologus 1998) } \\
\text { Beobachtungen III = Beobachtungen zu den Thuky- } \\
\text { didesscholien III (aus dem Nachlaß herausgegeben } \\
\text { von Klaus Alpers), Philologus 155, 2011, 257-271 } \\
\text { Beobachtungen zu einigen 'recentiores' des Thuky- }\end{array}$ \\
\hline
\end{tabular}


Kominis

La paléographie grecque $=$ et byzantine

Latte, Proll.

LGM

Luschnat, RE

Luschnat, Thuc. $\mathrm{I}^{2}$

Luschnat, Philologus 1954 / Schol. /

Thuk.scholien

Luzzatto, Itinerari

Nilsson

Omont, Inventaire

Philologus 142

Philologus 1964

Philologus 1998

Piccirilli, Storie

Powell, Aldine Scholia

Powell, The Archetype

Reitzenstein, Anfang

Reitzenstein, Gesch.

Rez. Alberti I

Rez. Alberti II dides, Heidelberg 1957 (= Beobachtungen zu einigen recc.)

Geschichte des Thukydidestextes im Mittelalter, Berlin 1965 (= Gesch.)

Rez. Alberti I, Gnomon 49, 1977, 754-773 (= Gnomon 1977)

Rez. Alberti II, Gnomon 70, 1998, 289-299

= Anasthasios K., Gregorio Pardo metropolita di Corinto e la sua opera, Roma 1960

La paléographie grecque et byzantine (Paris, 21-25 octobre 1974), Paris 1977

= Kurt L., Prolegomena, in: Hesychii Alexandrini Lexicon, ed. Kurt L., I, Hauniae 1953, VII-LI

= Lexica Graeca minora, selegit Kurt Latte, disposuit et praefatus est Harmut Erbse, Hildesheim 1965

= Otto L., Thukydides der Historiker, RE Suppl. XII, 1970, 1085-1354

$=$ siehe Thukydidesausgaben: Luschnat

$=$ Otto L., Die Thukydidesscholien. Zu ihrer handschriftlichen Grundlage, Herkunft und Geschichte, Philologus 98, 1954, 14-58

= Maria Jagoda L., Itinerari di codici antichi: un'edizione di Tucidide tra il II e il X secolo, MD 30, 1993, 167-203

= Martin P. N., Griechische Feste von religiöser Bedeutung mit Ausschluss der attischen, Leipzig 1906

= Henry O., Inventaire sommaire des manuscrits grecs de la Bibliothèque Nationale, II-III, Paris 1888

$=$ Beobachtungen II: siehe Kleinlogel

$=$ Beobachtungen I: siehe Kleinlogel

$=$ Beobachtungen II: siehe Kleinlogel

= Luigi P., Storie dello storico Tucidide, Genova 1985

$=$ J. Enoch P., The Aldine Scholia to Thucydides, CQ 30, 1936, 146-150

= J. Enoch P., The Archetype of Thucydides, CQ 32, 1938, 75-79

= Richard R., Der Anfang des Lexikons des Photios, Leipzig-Berlin 1907

= Richard R., Geschichte der griechischen Etymologika. Ein Beitrag zur Geschichte der Philologie in Alexandria und Byzanz, Leipzig 1897

= Gnomon 1977: siehe Kleinlogel

$=$ siehe Kleinlogel 


$\begin{array}{ll}\text { Richard, Répertoire }= & \text { Marcel R., Répertoire des bibliothèques et des cata- } \\ & \text { logues de manuscrits grecs, Paris 1958 } \\ \text { Schwabe, Att. / Attic. }= & \text { Ernst S., Aelii Dionysii et Pausaniae Atticistarum } \\ & \text { fragmenta, Lipsiae } 1890 \\ \text { Schwabe, Quaest. }= & \text { Ernst S., Quaestiones de scholiorum Thucydideo- } \\ & \text { rum fontibus, Leipz. Stud. z. cl. Philol. 4, 1881, } \\ & \text { } 5 \text {-150 } \\ = & \text { Aelii Dionysii Halicarnasensis reliquias coll. et ill. } \\ & \text { Carolus Th. Ph. S., Traiecti ad Rhenum 1877 } \\ = & \text { Sammlung griechischer und lateinischer Grammati- } \\ & \text { ker } \\ \text { Schwartz } & \text { Helmut v. Th., Scholia D in Iliadem secundum co- } \\ & \text { dices manu scriptos, 2000, <http://kups.ub.uni- } \\ \text { SGLG } & \text { koeln.de/volltexte/2006/1810> } \\ = & \text { Leslie T., The Grammar of Attic Inscriptions, I, Ber- } \\ & \text { lin-New York 1980 } \\ \text { van Thiel }= & \text { Renzo T., Hesychiana, MCr 10/12, 1975/7, 293-301 } \\ = & \text { Renzo T., Marginalia lexicographica, MCr 10/12, } \\ & 1975 / 7,369-374 \\ \text { Threatte }= & \text { Renzo T., L'ottavo libro di Tucidide nella lessicogra- } \\ & \text { fia, MCr 18, 1983, 161-192 } \\ \text { Tosi, Hes. II }= & \text { Renzo T., Studi sulla tradizione indiretta dei classici } \\ \text { Tosi, MCr X/XII / } & \text { greci, Bologna 1988 } \\ \text { Marginalia } & \text { Georg W., Beiträge zur Geschichte der griechischen } \\ \text { Tosi, Ott. libro } & \text { Lexikographen, Sitzungsb. der kgl. preuss. Akad. der } \\ & \text { Wissensch. zu Berlin 1895, 477-487 (= LGM 1-11) } \\ \text { Tosi, Studi } & \text { Carlo O. Z., Il Trattato di Gregorio Corinzio sull' } \\ & \text { Atticismo, Atti della Reale Accad. di Torino 27, } \\ & 1891 / 2,572-592 \\ \text { Wentzel, Beiträge } & \end{array}$

\section{Thukydidesausgaben}

$\begin{array}{ll}\text { Alberti }= & \text { Giovan B. A., Thucydidis Historiae, I-III, Romae } \\ & 1972-2000 \\ = & \text { Thucydides, ed. Aldus Manutius, Venetiis } 1502 \\ \text { Aldina } & \text { siehe Gottleber-Bauer-Beck } \\ \text { Bauer } & \text { Thucydidis De bello Peloponnesiaco libri octo, rec. } \\ \text { Bekker } & \text { Immanuel B., accedunt scholia Graeca et Dukeri } \\ & \text { Wassiique annotationes, I-IV, Berolini 1821 } \\ = & \text { Thucydides cum scholiis et antiquis et utilibus sine } \\ & \text { quibus autor intellectu multum est difficilis. Acces- } \\ & \text { sit praeterea diligentia Ioachimi C., in castigando } \\ \text { Camerarius } & \text { tum textu, tum commentariis una cum annotationi- }\end{array}$




\begin{tabular}{|c|c|c|}
\hline & & $\begin{array}{l}\text { bus eius }[\ldots] \text {, Basileae [ex officina Hervagiana] } \\
1540\end{array}$ \\
\hline Classen-Steup (Cl.-St.) & $=$ & $\begin{array}{l}\text { Thukydides, erkl. v. Johannes C., bearb. v. Julius S., } \\
\text { I, Berlin 51919; II, Berlin 51914; III-VIII, Berlin } \\
\text { 1892-31922 }\end{array}$ \\
\hline Didotausgabe & $=$ & 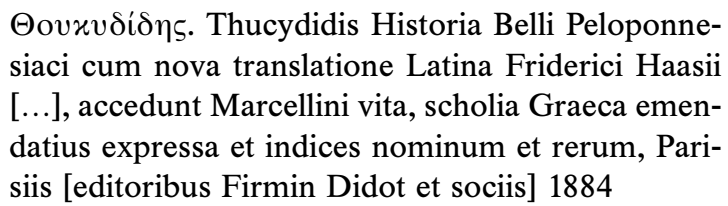 \\
\hline Duker & $=$ & $\begin{array}{l}\text { Thucydidis de bello Peloponnesiaco libri octo, ed. } \\
\text { Carolus A. D., Amstelaedami } 1731 \text { (Glasgow } 21759 \text { ) }\end{array}$ \\
\hline Gail & $=$ & $\begin{array}{l}\text { Jean-Baptiste G., Histoire Grecque de Thucy- } \\
\text { dide [...], I, Paris } 1807, \text { S. } 2 \text { (Variantes) }\end{array}$ \\
\hline Gottleber-Bauer-Beck & $=$ & $\begin{array}{l}\text { Thucydidis Olori fil. de bello Peloponnesiaco libri } \\
\text { VIII, ad editionem C. A. Duckeri cum omnibus } \\
\text { auctariis recusi accesserunt variae lectiones duorum } \\
\text { codicum animadversiones Ioannis C. Gottleberi [...] } \\
\text { coeptum opus perfecit suas notas adiecit indicem } \\
\text { Duckeri et glossarium Gottleberi auxit Carolus L. } \\
\text { Bauerus, Lipsiae } 1790 \text { (libri I-V); Thucydidis } \\
\text { Olori fil. de bello Peloponnesiaco libri VIII, ad } \\
\text { editionem C. A. Duckeri cum omnibus auctariis } \\
\text { recusi accesserunt variae lectiones duorum codi- } \\
\text { cum animadversiones Ioannis C. Gottleberi [...] et } \\
\text { C. L. Baueri [...] editionem absolvit Christianus D. } \\
\text { Beckius, volumen secundum lib. VI-VIII auctaria } \\
\text { praefationes indices, Lipsiae } 1804\end{array}$ \\
\hline Hude & $=$ & $\begin{array}{l}\text { Scholia in Thucydidem ad optimos codices collata, } \\
\text { ed. Carolus H., Lipsiae } 1927\end{array}$ \\
\hline Hude, Ed. maxima & $=$ & $\begin{array}{l}\text { Thucydidis Historiae, ad optimos codices denuo } \\
\text { ab ipso collatos rec. Carolus H., I-II, Lipsiae } \\
\text { 1898-1901 }\end{array}$ \\
\hline Hude, Ed. maior & $=$ & $\begin{array}{l}\text { Thucydidis Historiae, rec. Carolus H., I-II, Lipsiae } \\
1901 \text { (iterum rec. C. H., I-II, Lipsiae 1913-1925) }\end{array}$ \\
\hline Hudson & $=$ & $\begin{array}{l}\text { Joannes H., Thucydidis de bello Peloponnesiaco li- } \\
\text { bri octo, Oxoniae } 1696\end{array}$ \\
\hline Luschnat & $=$ & $\begin{array}{l}\text { Thucydidis Historiae, post Carolum Hude ed. Otto } \\
\text { L., I (libri I-II), editio altera correctior, Leipzig } 1960\end{array}$ \\
\hline Poppo & $=$ & $\begin{array}{l}\text { Thucydidis De bello Peloponnesiaco libri octo, ed. } \\
\text { Ernestus F. P., I-IV (11 voll.), Lipsiae 1821-1840 }\end{array}$ \\
\hline de Romilly & $=$ & $\begin{array}{l}\text { Thucydide. La guerre du Péloponnèse. Livre I, texte } \\
\text { ét. et trad. par Jacqueline d. R., Paris } 1953\end{array}$ \\
\hline
\end{tabular}


Stephanus

$=$ Thucydidis de bello Peloponnesiaco libri VIII, iidem Latine, ex interpretatione Laurentii Vallae, ab Henrico S. recognita [...], excudebat H. S., Genevae 1564 (21588)

\section{Verzeichnis abgekürzter Literaturangaben in den Apparaten}

\begin{tabular}{|c|c|c|}
\hline Abresch, Diluc. & $=$ & $\begin{array}{l}\text { Friedrich L. A., Dilucidationes Thucydideae, I-III, } \\
\text { Traiecti a. R. 1753-1763 }\end{array}$ \\
\hline Altinger & $=$ & $\begin{array}{l}\text { Friedrich A., De rhetoricis in orationes Thucydi- } \\
\text { deas scholiis, Monachii } 1885\end{array}$ \\
\hline Andrewes(, H. C.) & $=$ & siehe Gomme-Andrewes[-Dover] \\
\hline Beloch & $=$ & $\begin{array}{l}\text { Karl J. B., Griechische Geschichte, Berlin-Leipzig } \\
21923\end{array}$ \\
\hline Boread. & $=$ & 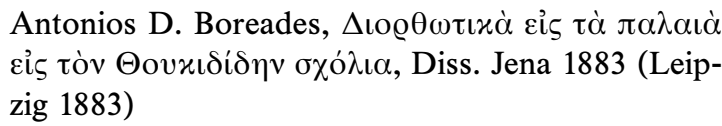 \\
\hline Bühler, Beiträge & $=$ & $\begin{array}{l}\text { Winfred B., Beiträge zur Erklärung der Schrift vom } \\
\text { Erhabenen, Göttingen } 1964\end{array}$ \\
\hline \multirow[t]{2}{*}{ Busolt-Swoboda } & $=$ & $\begin{array}{l}\text { Georg B.(-Heinrich S.), Griechische Staatskunde, I, } \\
\text { München } 1920\end{array}$ \\
\hline & & $\begin{array}{l}\text { Darstellung einzelner Staaten und der zwischen- } \\
\text { staatlichen Beziehungen, bearb. v. Heinrich S., } \\
\text { München } 1926\end{array}$ \\
\hline Cohn, Plato-Schol. & $=$ & $\begin{array}{l}\text { Leopold C., Untersuchungen über die Quellen der } \\
\text { Plato-Scholien, Jahrb. f. cl. Philol. Suppl. 13, 1884, } \\
\text { 773-864 }\end{array}$ \\
\hline Delachaux & $=$ & $\begin{array}{l}\text { Albert D., Notes critiques sur Thucydide, Neuchâ- } \\
\text { tel } 1925\end{array}$ \\
\hline $\begin{array}{l}\text { Deubner, Att. } \\
\text { Feste }\end{array}$ & $=$ & Ludwig D., Attische Feste, Berlin 1932 \\
\hline Dimitropoulos & $=$ & $\begin{array}{l}\text { Panagiotis D., Untersuchungen zum finalen Genetiv } \\
\text { des substantivierten Infinitivs bei Thukydides, Hel- } \\
\text { sinki } 1999\end{array}$ \\
\hline Doberentz & $=$ & $\begin{array}{l}\text { Emil D., De scholiis in Thucydidem commentatio, } \\
\text { Halis Saxonum } 1875\end{array}$ \\
\hline Dobree, Adv. & $=$ & Petri P. D. Adversaria [...], I, London 1883 \\
\hline Dover, H. C. & $=$ & siehe Gomme-Andrewes[-Dover] \\
\hline $\begin{array}{l}\text { Dover, Popular } \\
\quad \text { Morality }\end{array}$ & $=$ & $\begin{array}{l}\text { Kenneth J. D., Greek Popular Morality in the Time } \\
\text { of Plato and Aristotle, Oxford } 1974\end{array}$ \\
\hline
\end{tabular}


Egenolff, Orth.

Egenolff, Orthoep.

Ehlert

Erbse, vol. II / vol. III

Gomme, Essays

Gomme-Andrewes[Dover]

Goslings (Gosl.)

Hammond, Epirus

Hill, Sources

Judeich, Topogr.

Kirchner, Prosop. Att.

Kromayer-Veith

Lehrs, Ar. ${ }^{3}$

Lobeck, Phryn.

Loraux, Invention

Luschnat, Fschr. Teubner
$=$ Peter E., Die orthographischen Stücke der byzantinischen Litteratur, Leipzig 1888

$=$ Peter E., Die orthoepischen Stücke der byzantinischen Litteratur, Leipzig 1887

= Johannes E., De verborum copia Thucydidea quaestiones selectae, Diss. Berlin 1910

$=$ siehe Erbse, Bd. II / Bd. III

$=$ Arnold W. G., Essays in Greek History and Literature, Oxford 1937

= Arnold W. G.-Antony A.-Kenneth J. D., A Historical Commentary on Thucydides, IV-V, Oxford 1970-1981

= Frans G., Observationes ad scholia in Thucydidem, Lugduni Batavorum 1874

$=$ Nicholas G. L. H., Epirus: The Geography, The Ancient Remains, The History and Topography of Epirus and Adjacent Areas, Oxford 1967

Hammond, Hist. Mac. I = Nicholas G. L. H., A History of Macedonia, I, Oxford 1972

$=$ Kulturpflanzen und Haustiere in ihrem Übergang aus Asien nach Griechenland und Italien sowie in das übrige Europa, historisch-linguistische Skizzen v. Victor H., achte Auflage neu hrsg. v. Otto S. [...], Berlin 1911

$=$ George F. H., Sources for Greek History between the Persian and Peloponnesian Wars, Oxford 1962

= Walther J., Topographie von Athen, München ${ }^{2} 1931$

= Iohannes K., Prosopographia Attica, I, Berlin 1901

= Johannes K.-Georg V., Heerwesen und Kriegführung der Griechen und Römer, München 1963

= Karl L., De Aristarchi studiis Homericis, Lipsiae 31882

$=$ Phrynichi eclogae nominum et verborum Atticorum [...] edidit, explicuit Christian A. L., Lipsiae 1820

$=$ Nicole L., L'invention d'Athènes. Histoire de l'oraison funèbre dans la «cité classique», Paris 1981

= Otto L., Beobachtungen zu den Thukydidesscholien, in: Miscellanea critica. Aus Anlaß des 150jährigen Bestehens der Verlagsgesellschaft und des Graphischen Betriebes B. G. Teubner, hrsg. v. den Mitgliedern der Redaktion der Bibliotheca Teubneriana Johannes Irmscher-Bruno Doer-Ursula Peters-Reimar Müller, I, Leipzig 1964, 122-128 
Madvig, Adv. crit.

Maurer

Müller, Dor.

Nickau

Nickau, Praef.

Oomen

Petrova

Pickard-Cambridge

Philippson-Kirsten

Reiske

Riepl

Ros

Schmid, Att.

Steup

Theodoridis, Philox.

Theodoridis, vol. II

Tosi, Hes. I

Tosi, Hes. III

Tosi, Note

Tosi, Scoli

Travlos
= Johan N. M., Adversaria critica ad scriptores Graecos et Latinos, I, Hauniae 1871

= Karl M., Interpolation in Thucydides, Leiden-New York-Köln 1995

$=$ Karl O. M., Die Dorier, II, Breslau 1824

$=$ Klaus N., Untersuchungen zur textkritischen Methode des Zenodotos von Ephesos, Berlin-New York 1977

= Ammonii qui dicitur liber de adfinium vocabulorum differentia, ed. Klaus N., Lipsiae 1966

$=$ Gerhard O., De Zosimo Ascalonita atque Marcellino, Diss. Monasteri Westphalorum 1926

= Daniela P., Das Lexikon „Über die Syntax“. Untersuchung und kritische Ausgabe des Lexikons im Codex Paris. Coisl. Gr. 345, Wiesbaden 2006

= Arthur P.-C., The Dramatic Festivals of Athens, Oxford ${ }^{2} 1968$

= Alfred P.-Ernst K., Die griechischen Landschaften, I, Frankfurt a.M. 1950; II, Frankfurt a. M. 1956

= Johann J. R., Animadversionum ad Graecos auctores volumen tertium quo Thucydides, Herodotus et Aristides pertractantur, Lipsiae 1761

= Wolfgang R., Das Nachrichtenwesen des Altertums mit besonderer Rücksicht auf die Römer, LeipzigBerlin 1913

= Joannes G. A. R., Die METABO $\Lambda$ H (Variatio) als Stilprinzip des Thukydides, Nijmegen 1938

$=$ Wilhem Sch., Der Atticismus in seinen Hauptvertretern. Von Dionysius von Halikarnass bis auf den zweiten Philostratus, III-IV, Stuttgart 1893-1896

$=$ siehe Classen-Steup

$=$ Die Fragmente des Grammatikers Philoxenos, ed. Christos Th., Berlin-New York 1976

= Photii Patriarchae Lexicon, ed. Christos Th., II, Berlin-New York 1998

= Renzo T., Hesychiana, MCr 8/9, 1973/4, 252-260

= Renzo T., Hesychiana, MCr 13/14, 1978/9, 455-460

$=$ Renzo T., Note a Tucidide, MCr 10/12, 1975/7, 155-163

= Renzo T., Scoli-fantasma tucididei, Pisa 1983

$=$ John T., Bildlexikon zur Topographie des antiken Athen, Tübingen 1971 
Valk

Vitalis 1930

Voltz

Wachsmuth, Alterthumsk.

Wyse

Zahrnt
$=$ Eustathii Archiepiscopi Thessalonicensis Commentarii ad Homeri Iliadem pertinentes, ed. Marcus v. d. V., I-IV, Lugduni Batavorum 1971-1987

$=$ Gerhard V., Die Entwicklung der Sage von der Rückkehr der Herakliden [...], Diss. Greifswald 1930

= Ernst V., Die Thukydidespapyri, Diss. Straßburg i. E. 1911

= Wilhelm W., Hellenische Alterthumskunde aus dem Gesichtspunkte des Staates, I, Halle 21846

$=$ William W., The Speeches of Isaeus, Cambridge 1904

$=$ Michael Z., Olynth und die Chalkidier. Untersuchungen zur Staatenbildung auf der Chalkidischen Halbinsel im 5. und 4. Jahrhundert v. Chr., München 1971

\section{Verzeichnis abgekürzter Namen in den Apparaten}

Ald.

s. Aldina

Bau.

s. Bauer

Bip.

$=$ Editio Bipontina (Thucydidis de bello Peloponnesiaco libri octo, I-V, Zweibrücken 1788-1789)

Bloomf.

$=$ Samuel T. Bloomfield, The History of Thucydides, I-III, London 1829

Bk.

s. Bekker

Charit.

s. C. Charitonidis

Duk.

s. Duker

Goell.

$=$ Franz Göller, Thucydidis De bello Peloponnesiaco libri octo, I-II, Lipsiae 1836

Gosl.

s. Goslings

Gottl.

s. Gottleber

Gramm.

$=$ H. Gram (vd. Einleitung S. 44 Anm. 14)

Herw.

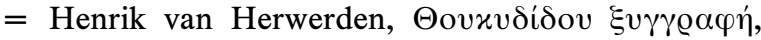
I-V, Traiecti a. R. 1877-1882

$\mathrm{Hu}$.

s. Hude

Huds.

s. Hudson

$\mathrm{Kr}$.

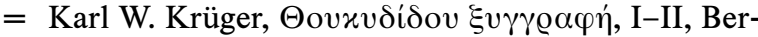
lin 1860

Pop.

s. Poppo 
Port.

$=$ Thucydidis Olori filii De bello Peloponnesiaco libri octo, iidem Latine, ex interpretatione Laurentii Vallae, ab Henrico Stephano recognita. Quam Aemilius Portus, Francisci P[orti] cr. f. paternos commentarios accurate sequutus ab infinita grauissimorum errorum multitudine nouissime repurgauit, magnaque diligentia passim expolitam innouauit, Francofurti 1594

Steph.

s. Stephanus 
